\title{
Modulation Instability, Breathers, and Bound Solitons in an Erbium-Doped Fiber System with Higher-Order Effects
}

\author{
Rui Guo, ${ }^{1}$ Hui-Qin Hao, ${ }^{1}$ and Xiao-Song Gu$^{2}$ \\ ${ }^{1}$ School of Mathematics, Taiyuan University of Technology, Taiyuan 030024, China \\ ${ }^{2}$ PetroChina Kunlun Gas Co., Ltd., Beijing 100101, China \\ Correspondence should be addressed to Hui-Qin Hao; math0351@sina.com
}

Received 8 November 2013; Accepted 17 February 2014; Published 6 April 2014

Academic Editor: Jaume Giné

Copyright (c) 2014 Rui Guo et al. This is an open access article distributed under the Creative Commons Attribution License, which permits unrestricted use, distribution, and reproduction in any medium, provided the original work is properly cited.

We mainly investigate the generalized nonlinear Schrödinger-Maxwell-Bloch system which governs the propagation of optical solitons in nonlinear erbium-doped fibers with higher-order effects. We deduce Lax pair, analyze modulation instability conditions, construct the Darboux transformation, and derive the Akhmediev breathers, Ma-breathers, bound solitons, and two-breather solutions for this system. Considering the influences of higher-order effects, propagation properties of those solitons are discussed.

\section{Introduction}

In recent years, optical solitons have attracted many researchers for their potential applications in optical fiber transmission systems [1-3]. Based on the balance of the self-phase modulation and group velocity dispersion, the propagation of optical solitons in the picosecond regime is usually governed by the nonlinear Schrödinger [4] equation

$$
i q_{z}+q_{t t}+2|q|^{2} q=0
$$

where $q$ denotes the slowly varying complex envelope of the wave. When considering propagation characters of the ultrashort pulses, (1) cannot describe the corresponding physical mechanism due to the absences of the fourth-order dispersion, higher-order nonlinearities, and self-steepening effects. Owing to the above three factors, the dynamic features of the ultrashort pulses can be depicted by the following generalized nonlinear Schrödinger equation (GNLS) $[5,6]$ :

$$
\begin{aligned}
& i q_{z}+q_{t t}+2|q|^{2} q \\
& +\tau\left(q_{t t t t}+8|q|^{2} q_{t t}+2 q^{2} q_{t t}^{*}+6 q^{*} q_{t}^{2}\right. \\
& \left.\quad+4\left|q_{t}\right|^{2} q+6|q|^{4} q\right)=0,
\end{aligned}
$$

where $\tau$ is a small dimensionless real parameter, and it is usually positive. In addition, (2) can also govern the nonlinear spin excitations in one-dimensional isotropic biquadratic Heisenberg ferromagnetic spin with the octupole-dipole interaction $[7,8]$.

In real optic fibers, the attenuation usually exists, in this context, erbium-doped fibers can minimize the attenuation [9]. The mathematical description of solitons propagating in erbium-doped fibers is the nonlinear Schrödinger-MaxwellBloch (NLS-MB) equations [10, 11]:

$$
\begin{gathered}
q_{z}=i\left(\frac{1}{2} q_{t t}+|q|^{2} q\right)+2 p, \\
p_{t}=2 i \omega p+q \eta, \\
\eta_{t}=-\left(q p^{*}+p q^{*}\right),
\end{gathered}
$$

where subscripts $z$ and $t$ denote the partial derivatives with respect to the longitudinal distance and retarded time. $\omega$ is the frequency, the asterisk denotes the complex conjugate, $p=v_{1} v_{2}^{*}$, and $\eta=\left|v_{2}\right|^{2}-\left|v_{1}\right|^{2}$ with $v_{1}$ and $v_{2}$ representing the wave functions in a two-level system [12, 13]. Many research achievements about system (3a), (3b), and (3c) have been obtained [14-16].

However, when taking the effects such as the fourth-order dispersion, higher-order nonlinearities, and self-steepening effects into account, the propagation of optical solitons in fibers doped with two-level resonant impurities like erbium 


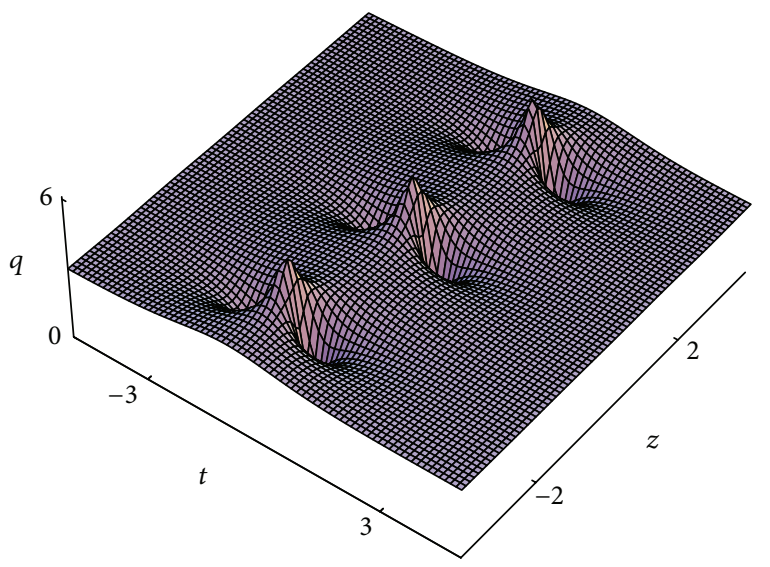

(a)

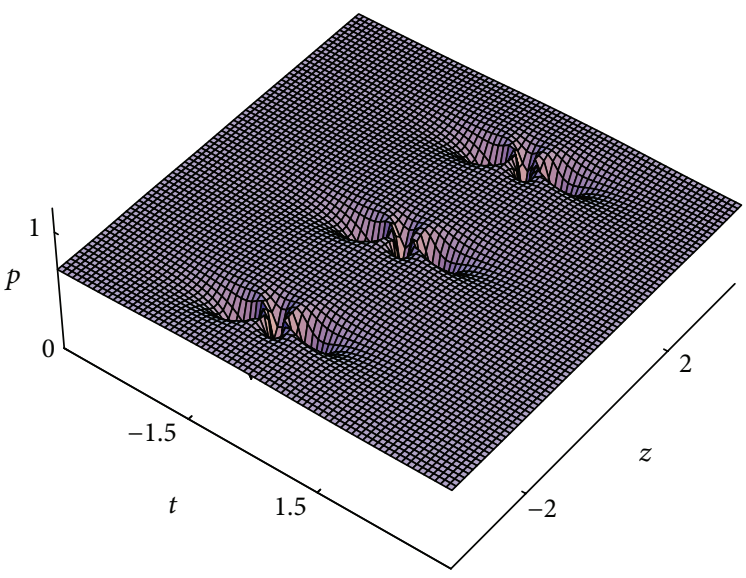

(b)

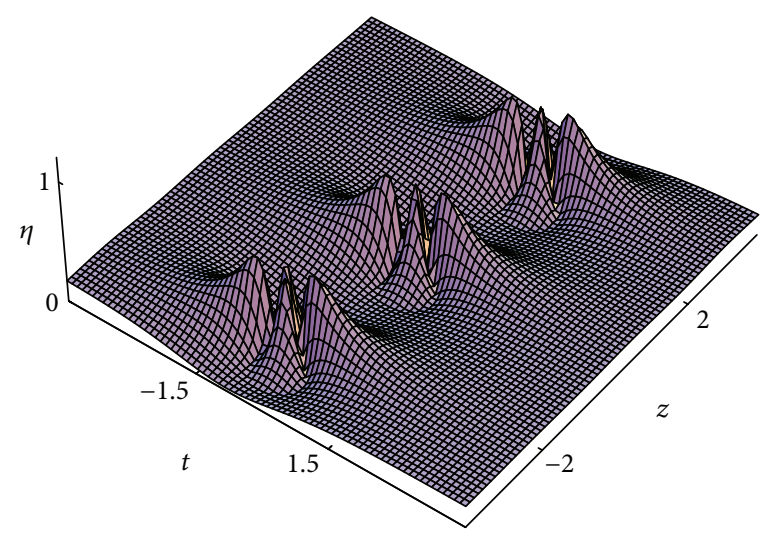

(c)

FIGURE 1: The parameters adopted here are $\kappa_{s}=2.3, q_{c}=1, \kappa_{c}=3, \omega=1$, and $\tau=0$.

is usually described by the following generalized nonlinear Schrödinger-Maxwell-Bloch (GNLS-MB) system [9]:

$$
\begin{gathered}
q_{z}=i\left(q_{t t}+2|q|^{2} q\right) \\
+i \tau\left(q_{t t t t}+8|q|^{2} q_{t t}\right. \\
+2 q^{2} q_{t t}^{*}+6 q^{*} q_{t}^{2}+4\left|q_{t}\right|^{2} q \\
\left.+6|q|^{4} q\right)+2 p, \\
p_{t}=2 i \omega p+q \eta, \\
\eta_{t}=-\left(q p^{*}+p q^{*}\right) .
\end{gathered}
$$

To our knowledge, investigations on system (4a), (4b), and (4c) have not been reported, and the aim of this paper is mainly to investigate the modulation instability conditions, generate the breather and bound solutions, and discuss the dynamic behaviors of those solutions for system (4a), (4b), and $(4 \mathrm{c})$.

The outline of this paper will be as follows: in Section 2, we will derive Lax pair and analyze the modulation instability conditions for system (4a), (4b), and (4c). In Section 3, by using the Darboux transformation, we will construct two types of one-breather solutions: Akhmediev breathers and $\mathrm{Ma}$ breathers on the nonzero continuous wave $(\mathrm{cw})$ background. In Section 4, we will discuss analytically the interactions between neighboring bound solitons and twobreather solutions for system (4a), (4b), and (4c). Finally, our conclusions will be addressed in Section 5 .

\section{Lax Pair and Modulation Instability for System (4a), (4b), and (4c)}

Employing the Ablowitz-Kaup-Newell-Segur formalism [17], we can derive the Lax pair for system (4a), (4b), and (4c) as

$$
\begin{aligned}
& \Psi_{t}=U \Psi, \\
& \Psi_{z}=V \Psi,
\end{aligned}
$$

where $\Psi=\left(\psi_{1}, \psi_{2}\right)^{T}$ (T denotes the transpose of a matrix) and the matrices $U$ and $V$ have the form

$$
\begin{gathered}
U=-i \lambda U_{1}+U_{0}, \\
V=\lambda^{4} V_{4}+\lambda^{3} V_{3}+\lambda^{2} V_{2}+\lambda V_{1}+V_{0}+\frac{1}{\lambda+\omega} V_{-1},
\end{gathered}
$$




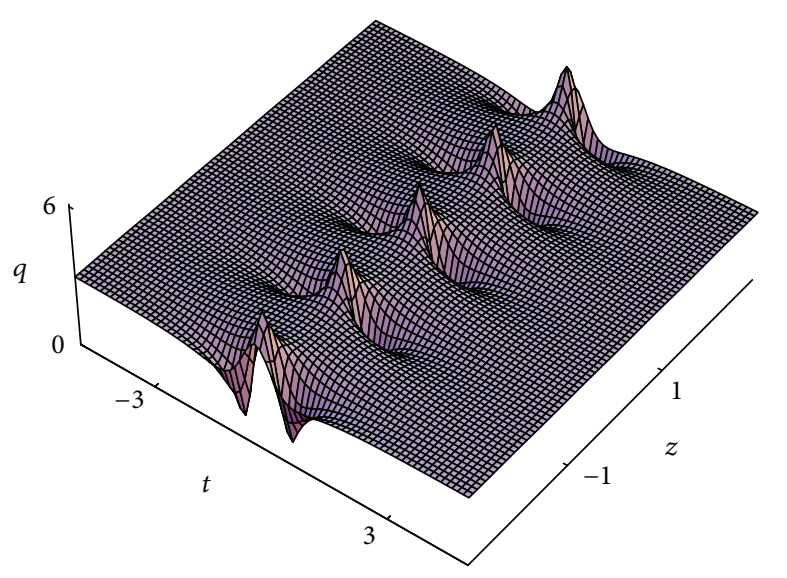

(a)

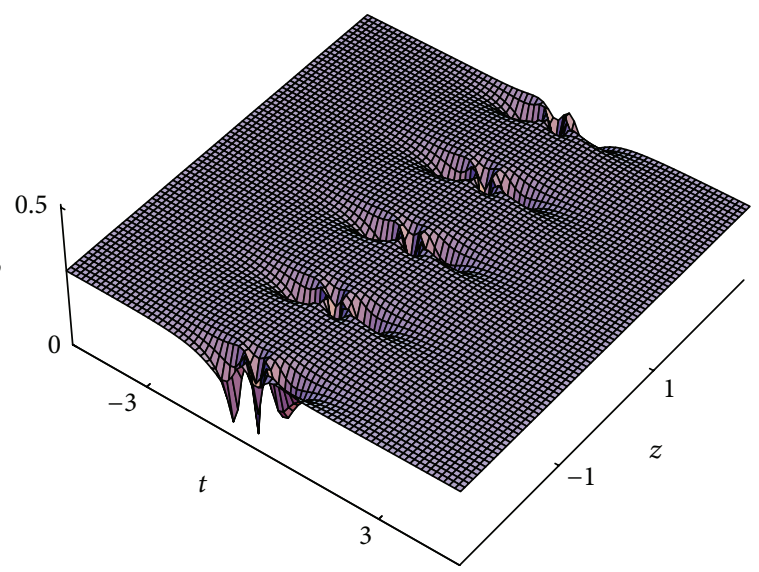

(b)

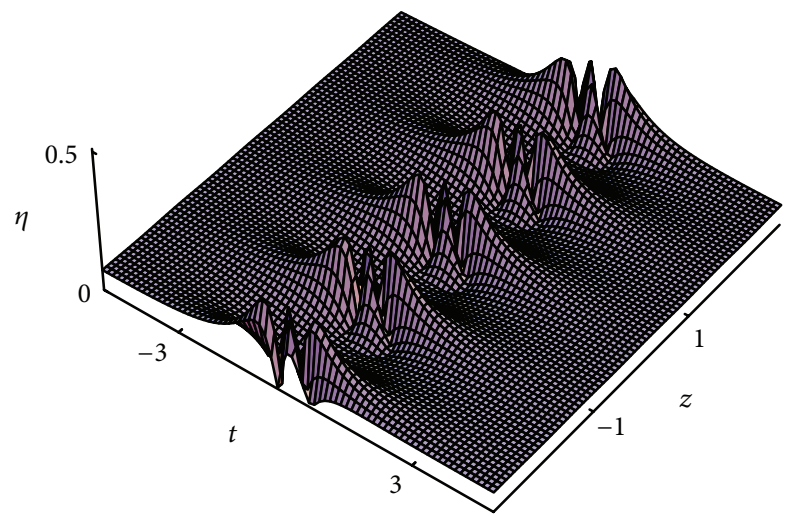

(c)

FIGURE 2: The parameters adopted here are $\kappa_{s}=2.3, q_{c}=1, \kappa_{c}=3, \omega=1$, and $\tau=0.1$.

with $\lambda$ as a spectral parameter,

$$
\begin{aligned}
& U_{1}=\left(\begin{array}{cc}
1 & 0 \\
0 & -1
\end{array}\right), \quad U_{0}=\left(\begin{array}{cc}
0 & q \\
-q^{*} & 0
\end{array}\right), \quad V_{4}=8 i \tau U_{1}, \\
& V_{2}=\left(\begin{array}{cc}
-2 i-4 i \tau|q|^{2} & -4 i \tau q_{t} \\
-4 i \tau q_{t}^{*} & 2 i+4 i \tau|q|^{2}
\end{array}\right), \quad V_{3}=-8 \tau U_{0}, \\
& V_{1}=\left(\begin{array}{cc}
2 \tau q^{*} q_{t}-2 \tau q q_{t}^{*} & 2 q+4 \tau|q|^{2} q+2 \tau q_{t t} \\
-2 q^{*}-4 \tau|q|^{2} q^{*}-2 \tau q_{t t}^{*} & -2 \tau q^{*} q_{t}+2 \tau q q_{t}^{*}
\end{array}\right), \\
& V_{0}=\left(\begin{array}{cc}
a_{11} & a_{12} \\
a_{21} & -a_{11}
\end{array}\right), \quad V_{-1}=i\left(\begin{array}{cc}
\eta & -p \\
-p^{*} & -\eta
\end{array}\right), \\
& a_{11}=i|q|^{2}+3 i|q|^{4}-i \tau\left|q_{t}\right|^{2}+i \tau q^{*} q_{t t}+i \tau q q_{t t}^{*}, \\
& a_{12}=i q_{t}+6 i \tau|q|^{2} q_{t}+i \tau q_{t t t}, \\
& a_{21}=i q_{t}^{*}+6 i \tau|q|^{2} q_{t}^{*}+i \tau q_{t t t}^{*} .
\end{aligned}
$$

Through symbolic computations, one can verify that system (4a), (4b), and (4c) can be concluded through the zero curvature equation $U_{z}-V_{t}+U V-V U=0$.

Modulational instability (MI), which results from the interplay between nonlinearity and dispersive effects, refers to a parametric process in which a continuous or quasicontinuous wave undergoes a modulation of its amplitude or phase in the presence of weak perturbations [18-20]. The MI has some applications in condensate physics, plasma physics, hydrodynamics, nonlinear optics, and some other branches of physics [18]. Now by introducing the steadystate $\mathrm{cw}$ solutions for system (4a), (4b), and (4c) as $q=$ $q_{0} \exp 2 i\left(\kappa+q_{0}^{2}+3 q_{0}^{4} \tau\right) z, p=i \kappa q_{0} \exp 2 i\left(k+q_{0}^{2}+3 q_{0}^{4} \tau\right) z$, $\eta=\omega \kappa$, here, with $p_{0}$ being the input power, we will discuss the modulation instability process for system (4a), (4b), and (4c).

We examine the MI process of the steady-state solutions by introducing the following perturbed solutions:

$$
\begin{gathered}
q=\left(q_{0}+A_{1}\right) \exp 2 i\left(\kappa+q_{0}^{2}+3 q_{0}^{4} \tau\right) z, \\
p=i\left(\kappa q_{0}+A_{2}\right) \exp 2 i\left(\kappa+q_{0}^{2}+3 q_{0}^{4} \tau\right) z, \\
\eta=\omega \kappa+B,
\end{gathered}
$$

where $A_{1}, A_{2}$, and $B$ are weak perturbations with the assumed general expressions being $A_{1}=u_{1} \cos (K z-\Omega t)+i v_{1} \sin (K z-$ $\Omega t), A_{2}=u_{2} \cos (K z-\Omega t)+i v_{2} \sin (K z-\Omega t), B=b \cos (K z-$ $\Omega t$ ), where $u_{1}, v_{1}, u_{2}, v_{2}$, and $b$ are real amplitudes of those perturbations, $\Omega$ denotes the real frequency of modulation 


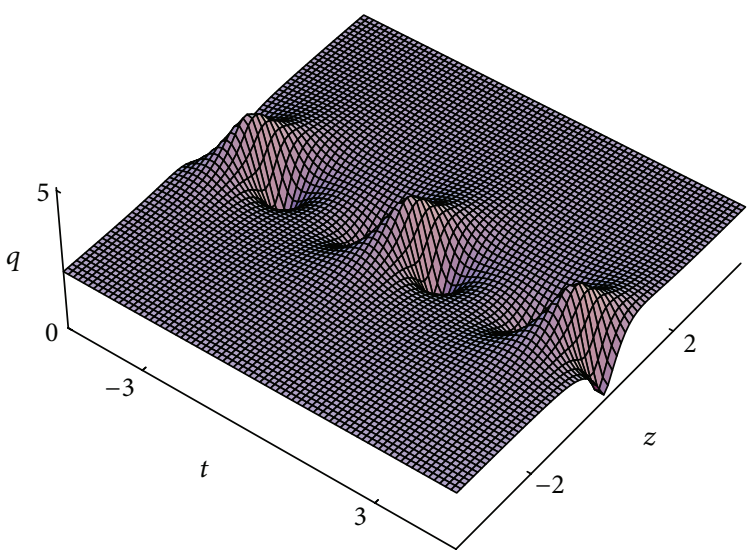

(a)

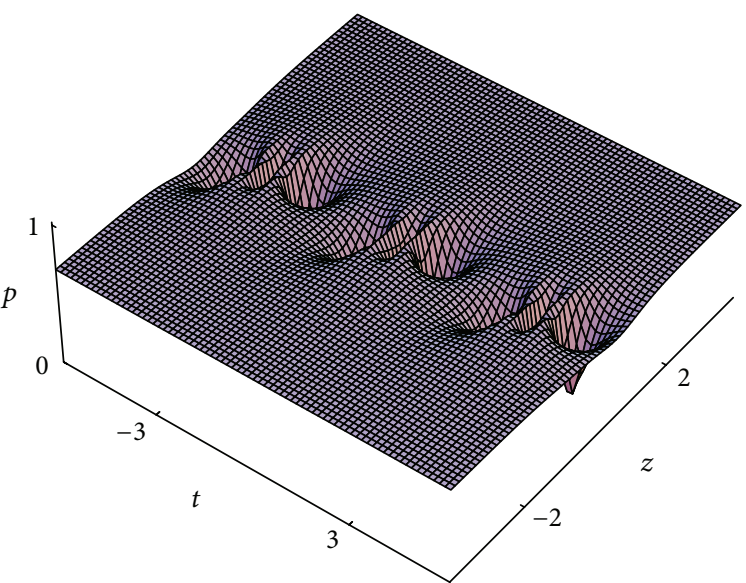

(b)

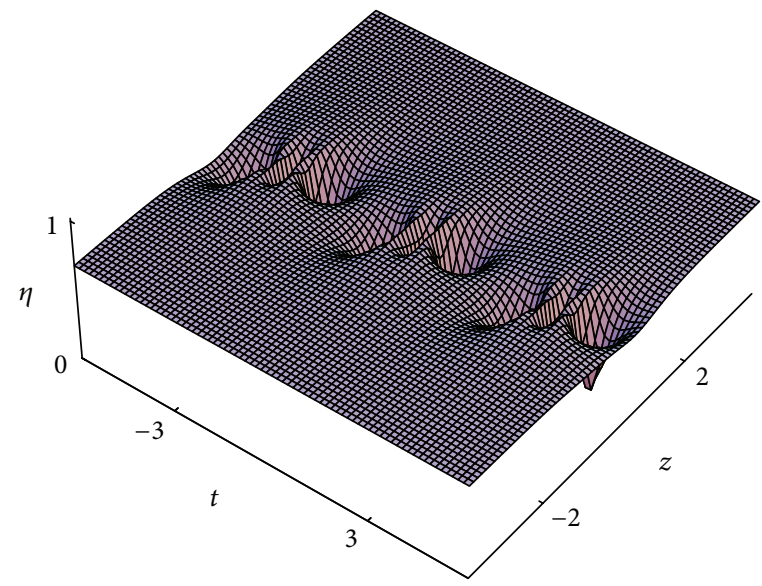

(c)

FIGURE 3: The parameters adopted here are $\kappa_{s}=1.3, q_{c}=1, \kappa_{c}=3, \omega=1$, and $\tau=0.1$.

perturbations, and $K$ is the real disturbance wave number. Inserting (8a), (8b), and (8c) into (4b) and (4c), we can obtain the linearized equations of $u_{1}, v_{1}, u_{2}, v_{2}$, and $b$, so $u_{2}, v_{2}$, and $b$ can be solved as follows:

$$
\begin{gathered}
u_{2}=\frac{2 \omega \kappa\left(2 u_{1} \omega-v_{1} \Omega\right)}{4 q_{0}^{2}+4 \omega^{2}-\Omega^{2}}, \\
v_{2}=\frac{2 \kappa\left(2 q_{0}^{2} v_{1}+2 v_{1} \omega^{2}-u_{1} \omega \Omega\right)}{4 q_{0}^{2}+4 \omega^{2}-\Omega^{2}}, \\
b=\frac{2 \kappa q_{0}\left(v_{1} \Omega-2 u_{1} \omega\right)}{4 q_{0}^{2}+4 \omega^{2}-\Omega^{2}} .
\end{gathered}
$$

Substituting (8a), (8b), (9a), and (9b) into (4a) and (4b), collecting the linear terms, we can derive two linear homogeneous equations for the perturbed unknown functions $u_{1}$ and $v_{1}$ :

$$
\begin{gathered}
\left(4 q_{0}^{2}-\Omega^{2}\right) y_{1} u_{1}+y_{2} v_{1}=0, \\
y_{2} u_{1}-\Omega^{2} y_{1} v_{1}=0,
\end{gathered}
$$

with

$$
\begin{gathered}
y_{1}=2 \kappa-\left(4 q_{0}^{2}+4 \omega^{2}-\Omega^{2}\right)\left(1+6 q_{0}^{2} \tau-\Omega^{2} \tau\right), \\
y_{2}=4 \kappa \omega \Omega+K\left(4 q_{0}^{2}+4 \omega^{2}-\Omega^{2}\right) .
\end{gathered}
$$

Equations (10a) and (10b) have nontrivial solutions if and only if the following determinant formed by the coefficients matrix vanishes, that is,

$$
\left|\begin{array}{cc}
\left(4 q_{0}^{2}-\Omega^{2}\right) y_{1} & y_{2} \\
y_{2} & -\Omega^{2} y_{1}
\end{array}\right|=0 .
$$

Equation (12) leads to the dispersion relation of $K$ and $\Omega$ which determines the modulation instability process of the steady-state $\mathrm{cw}$ solution as

$$
K=\frac{4 \kappa \omega \Omega+\left|\Omega y_{1}\right| \sqrt{\Omega^{2}-4 q_{0}^{2}}}{\Omega^{2}-4 q_{0}^{2}-4 \omega^{2}} .
$$

From (13), one can conclude that if $\Omega^{2}-4 q_{0}^{2}<0$, the value of $K$ will be complex; then the modulation instability will take place with $|\operatorname{Im} K|$ as the instability growth rate. So $\Omega^{2}-4 q_{0}^{2}<$ 0 is the condition of the modulation instability for system (4a), (4b), and (4c). 


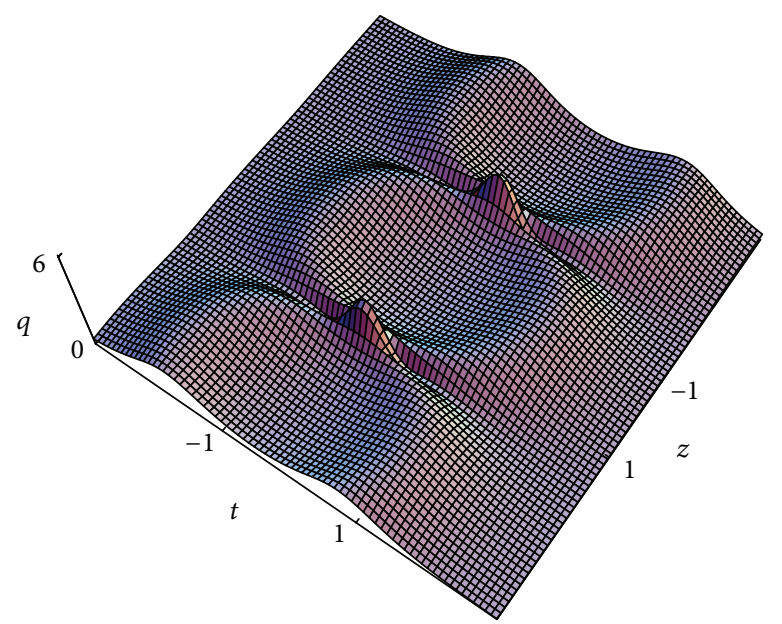

(a)

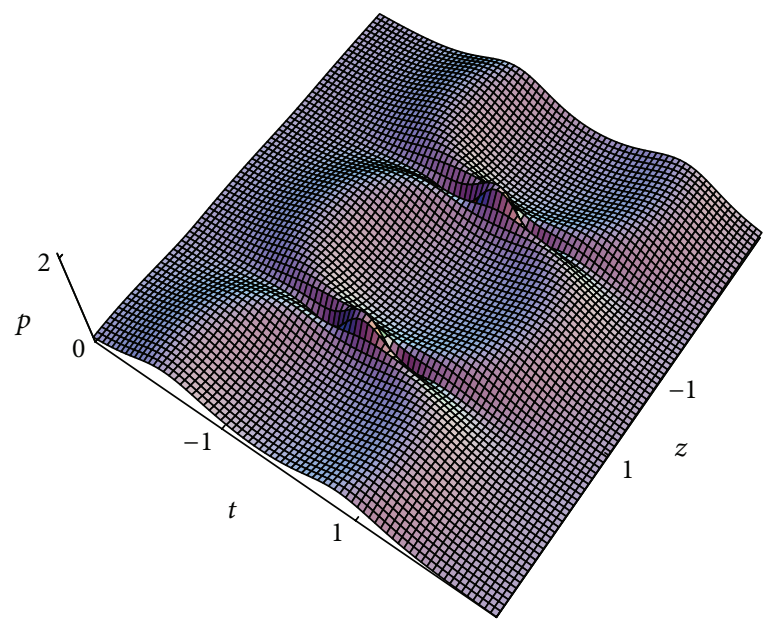

(b)

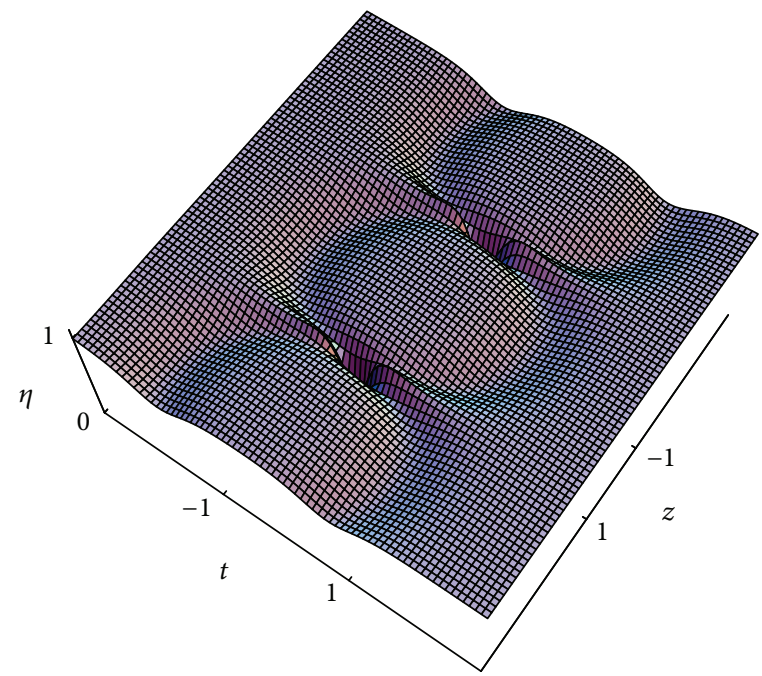

(c)

FIgURE 4: The parameters adopted here are $t_{0}=3, \omega=3$, and $\tau=0$.

\section{One-Breather Solutions for System (4a),} $(4 \mathrm{~b})$, and $(4 \mathrm{c})$

Based on Lax pair (5a) and (5b), we can construct the following Darboux transformation for system (4a), (4b), and (4c) as

$$
\begin{gathered}
q^{\prime}=q-2 i \frac{\left(\lambda_{1}-\lambda_{1}^{*}\right) \phi_{1} \phi_{2}^{*}}{\left|\phi_{1}\right|^{2}+\left|\phi_{2}\right|^{2}} \\
p^{\prime}=\frac{p \chi_{1}^{2}}{\chi_{2}\left(\left|\phi_{1}\right|^{2}+\left|\phi_{2}\right|^{2}\right)^{2}}-\frac{p^{*}\left(\lambda_{1}-\lambda_{1}^{*}\right) \phi_{1}^{2} \phi_{2}^{* 2}}{\chi_{2}\left(\left|\phi_{1}\right|^{2}+\left|\phi_{2}\right|^{2}\right)^{2}} \\
+\frac{2 \eta \chi_{1}\left(\lambda_{1}-\lambda_{1}^{*}\right) \phi_{1} \phi_{2}^{*}}{\chi_{2}\left(\left|\phi_{1}\right|^{2}+\left|\phi_{2}\right|^{2}\right)^{2}}, \\
\eta^{\prime}=\eta\left(1+\frac{2\left(\lambda_{1}-\lambda_{1}^{*}\right)\left|\phi_{1}\right|^{2}\left|\phi_{2}\right|^{2}}{\chi_{2}\left(\left|\phi_{1}\right|^{2}+\left|\phi_{2}\right|^{2}\right)^{2}}\right) \\
+\frac{p\left(\lambda_{1}-\lambda_{1}^{*}\right) \chi_{1} \phi_{2} \phi_{1}^{*}}{v_{n}\left(\left|\phi_{1}\right|^{2}+\left|\phi_{n}\right|^{2}\right)^{2}}-\frac{p^{*}\left(\lambda_{1}-\lambda_{1}^{*}\right) \chi_{1}^{*} \phi_{1} \phi_{2}^{*}}{v_{\lrcorner}\left(\left|\phi_{1}\right|^{2}+\left|\phi_{\wedge}\right|^{2}\right)^{2}}
\end{gathered}
$$

with

$$
\begin{gathered}
\chi_{1}=\left(\lambda_{1}+\omega\right)\left|\phi_{1}\right|^{2}+\left(\lambda_{1}^{*}+\omega\right)\left|\phi_{2}\right|^{2}, \\
\chi_{2}=\left(\lambda_{1}+\omega\right)\left(\lambda_{1}^{*}+\omega\right) .
\end{gathered}
$$

Considering the nonzero continuous wave background, we can take $q=q_{c} \exp i\left(\kappa_{c} z+\omega_{c} t\right), p=i p_{c} \exp i\left(\kappa_{c} z+\omega_{c} t\right)$, and $\eta=\eta_{c}$ as the initial seeds, where $q_{c}, p_{c}, \eta_{c}, \kappa_{c}$, and $\omega_{c}$ are all real parameters. System $(4 a),(4 b)$, and (4c) requires the following nonlinear dispersion relation:

$$
\begin{aligned}
p_{c}= & -\frac{1}{2}\left(2 q_{c}^{2}-\kappa_{c}-\omega_{c}^{2}+6 q_{c}^{4} \tau-12 q_{c}^{2} \omega_{c}^{2} \tau+\omega_{c}^{4} \tau\right), \\
\eta_{c}= & -\frac{1}{4}\left(2 \omega-\omega_{c}\right) \\
& \times\left(2 q_{c}^{2}-\kappa_{c}-\omega_{c}^{2}+6 q_{c}^{4} \tau-12 q_{c}^{2} \omega_{c}^{2} \tau+\omega_{c}^{4} \tau\right) .
\end{aligned}
$$




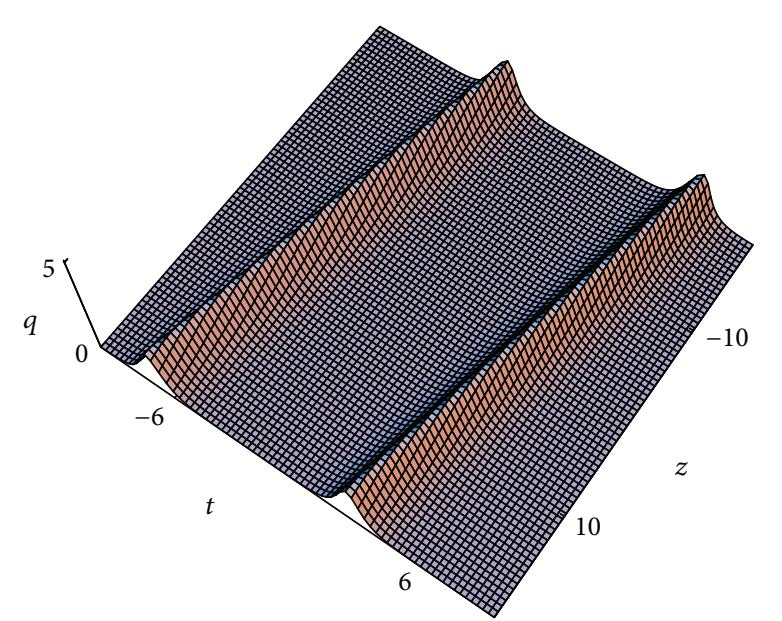

(a)

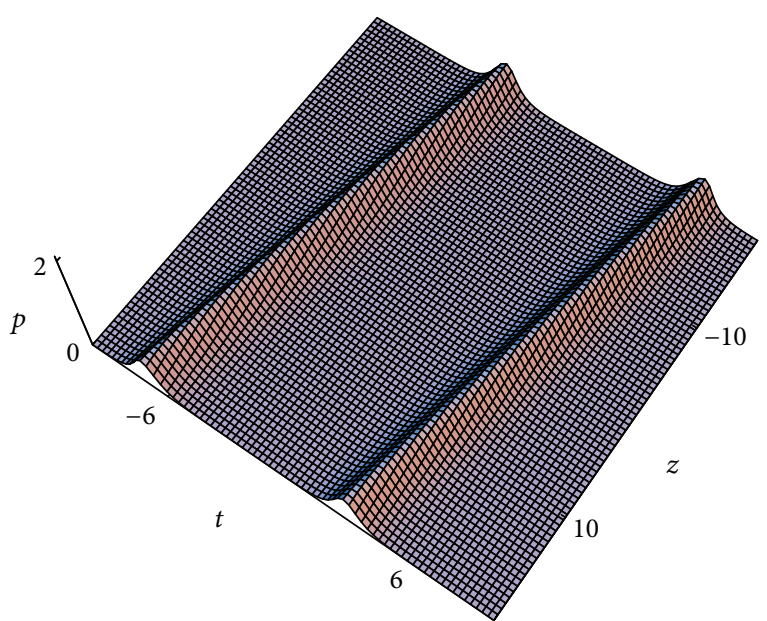

(b)

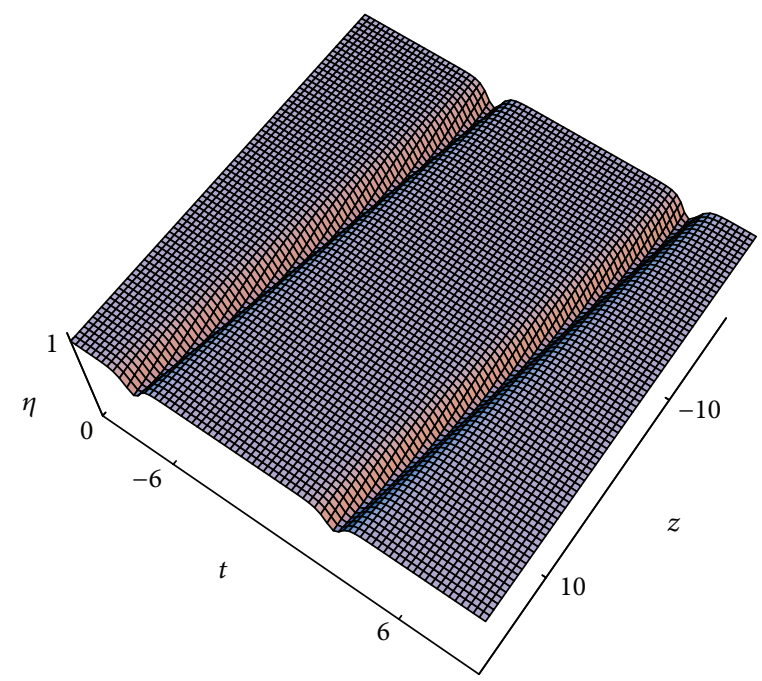

(c)

FIgURE 5: The parameters adopted here are $t_{0}=10, \omega=3$, and $\tau=0$.

By the method of separation of variables and the superposition principle, we can arrive at

$$
\begin{gathered}
\phi_{1}=\left(c_{1} \exp \theta_{1}+c_{2} \exp \theta_{2}\right) \exp i\left(\kappa_{c} z+\omega_{c} t\right), \\
\phi_{2}=c_{3} \exp \theta_{1}+c_{4} \exp \theta_{2},
\end{gathered}
$$

where

$$
\begin{aligned}
\theta_{1}= & \frac{t}{2}\left[-i \omega_{c}+\sqrt{-\omega_{c}^{2}-4 q_{c}^{2}-4 \lambda_{1}^{2}-4 \lambda_{1} \omega_{c}}\right] \\
& +\frac{z}{2}\left[-i \kappa_{c}+\sqrt{-\kappa_{c}^{2}+4 a^{2}-4 b^{2}-4 i a \kappa_{c}}\right], \\
\theta_{2}= & \frac{t}{2}\left[-i \omega_{c}-\sqrt{-\omega_{c}^{2}-4 q_{c}^{2}-4 \lambda_{1}^{2}-4 \lambda_{1} \omega_{c}}\right] \\
& +\frac{z}{2}\left[-i \kappa_{c}-\sqrt{-\kappa_{c}^{2}+4 a^{2}-4 b^{2}-4 i a \kappa_{c}}\right],
\end{aligned}
$$

with 


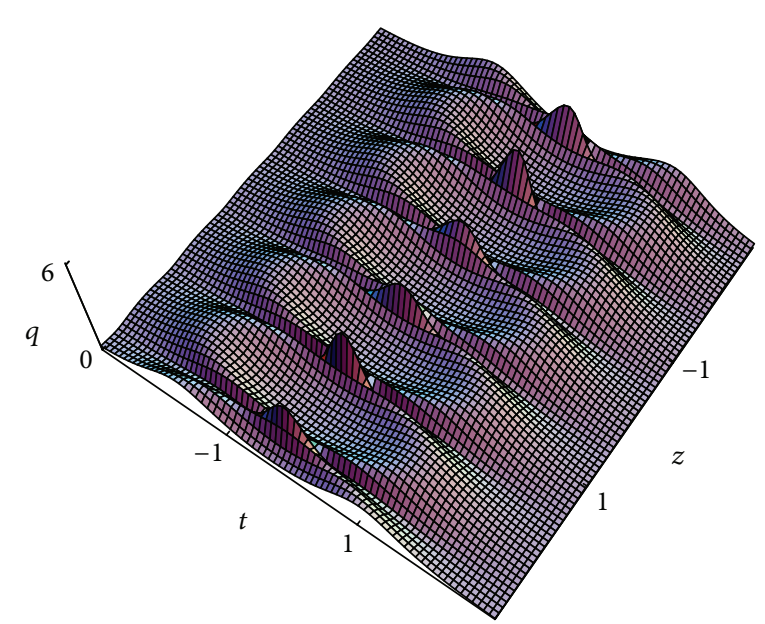

(a)

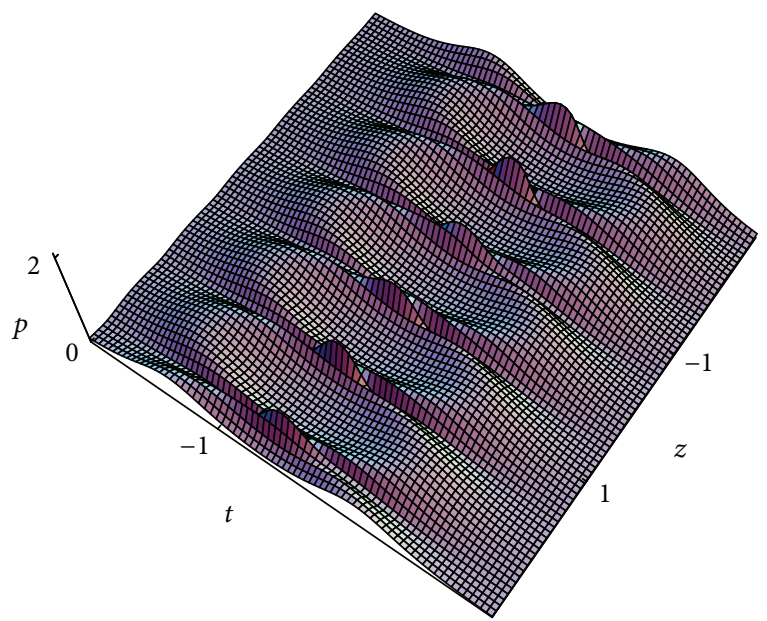

(b)

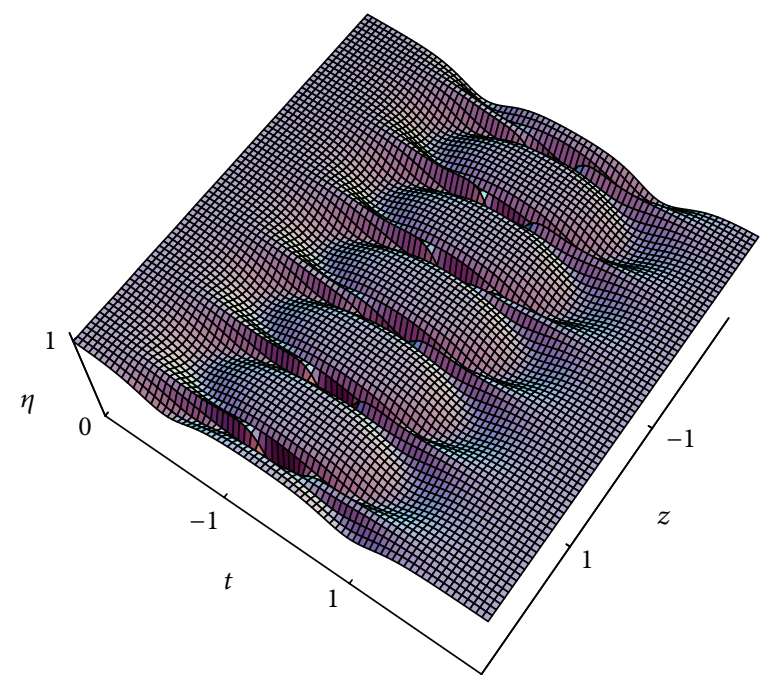

(c)

FIgURE 6: The parameters adopted here are $t_{0}=3, \omega=3$, and $\tau=5$.

and $c_{1}, c_{2}, c_{3}$, and $c_{4}$ are complex constants satisfied as

$$
\begin{aligned}
c_{3}= & \frac{1}{q_{c}}\left[i \omega_{c}+i \lambda_{1}+\frac{1}{2}\right. \\
& \left.\quad \times\left(-i \omega_{c}+\sqrt{-\omega_{c}^{2}-4 q_{c}^{2}-4 \lambda_{1}^{2}-4 \lambda_{1} \omega_{c}}\right)\right] c_{1}, \\
= & (L+i M) c_{1},
\end{aligned}
$$

$c_{2}$

$$
\begin{aligned}
& =\frac{q_{c}}{(1 / 2)\left(-i \omega_{c}+\sqrt{-\omega_{c}^{2}-4 q_{c}^{2}-4 \lambda_{1}^{2}-4 \lambda_{1} \omega_{c}}\right)+i \omega_{c}+i \lambda_{1}} c_{4} \\
& =(L+i M) c_{4} .
\end{aligned}
$$

Suppose that

$$
\begin{aligned}
& \lambda_{1}=\frac{1}{2}\left(A_{s}+i \kappa_{s}\right), \quad c_{4}=c_{1}, \\
& \zeta_{1}+i \eta_{1}=\sqrt{-\omega_{c}^{2}-4 q_{c}^{2}-4 \lambda_{1}^{2}-4 \lambda_{1} \omega_{c}} \\
& \zeta_{2}+i \eta_{2}=\sqrt{-\kappa_{c}^{2}+4 a^{2}-4 b^{2}-4 i a \kappa_{c}}
\end{aligned}
$$

with $A_{s}, \kappa_{s}, \zeta_{1}, \eta_{1}, \zeta_{2}$, and $\eta_{2}$ being real numbers. Now, substituting (17a) and (17b) into (14a), (14b), and (14c), we can obtain the solutions for system (4a), (4b), and (4c) as

$$
\begin{gathered}
q=\frac{G_{1}}{F_{1}} \exp i\left(\kappa_{c} z+\omega_{c} t\right), \\
p=\frac{G_{2}}{F_{2}} \exp i\left(\kappa_{c} z+\omega_{c} t\right), \\
\eta=\frac{G_{3}}{F_{2}},
\end{gathered}
$$




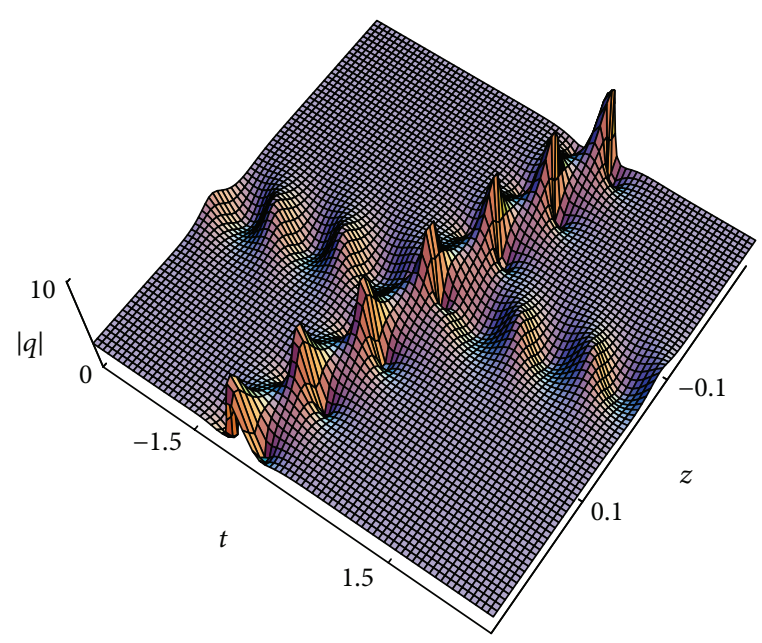

(a)

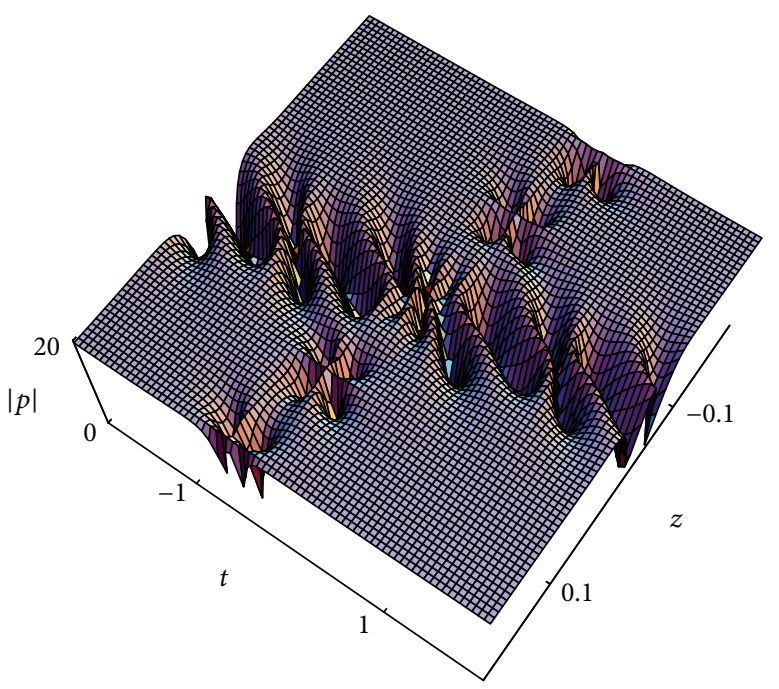

(b)

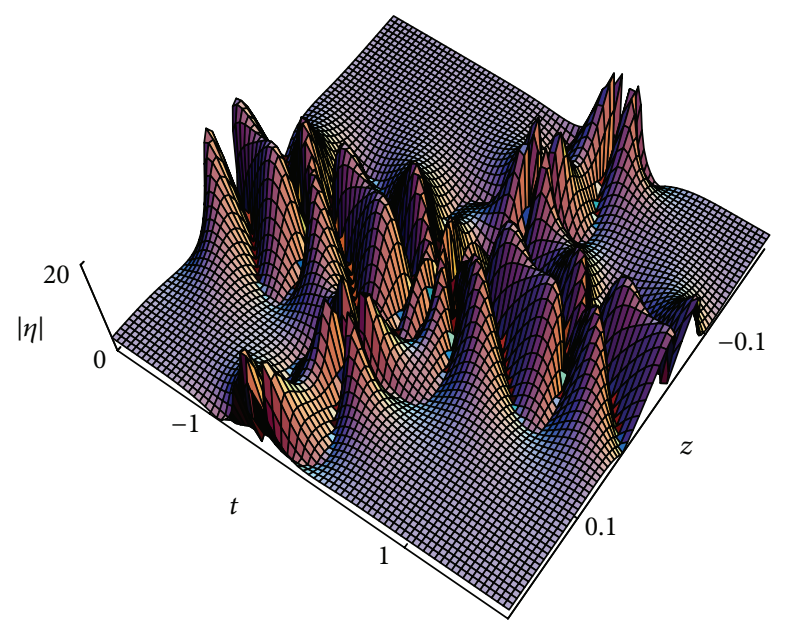

(c)

Figure 7: The parameters adopted here are $\lambda_{1}=i, \lambda_{2}=4 i, q_{c}=3, \omega_{c}=2, \kappa_{c}=1, \omega=0.5$, and $\tau=0.1$.

where

$$
\begin{aligned}
G_{1}= & q_{c}-2 i\left(\lambda_{1}-\lambda_{1}^{*}\right)\left[e^{\theta_{1}}+e^{\theta_{2}}(L+i M)\right] \\
& \times\left[e^{\theta_{2}^{*}}+e^{\theta_{1}^{*}}(L-i M)\right], \\
F_{1}= & e^{\theta_{1}^{*}}\left[2 L e^{\theta_{2}}+\left(1+L^{2}+M^{2}\right) e^{\theta_{1}}\right] \\
& +e^{\theta_{2}^{*}}\left[2 L e^{\theta_{1}}+\left(1+L^{2}+M^{2}\right) e^{\theta_{2}}\right], \\
G_{2}= & i p_{c}\left[\mu^{2}+\sigma_{1}^{2} \sigma_{2}^{* 2}\left(\lambda_{1}-\lambda_{1}^{*}\right)^{2}\right] \\
& +2 \eta_{0} \sigma_{1} \sigma_{2}^{*}\left(\lambda_{1}-\lambda_{1}^{*}\right) \mu, \\
F_{2}= & \left(\lambda_{1}+\omega\right)\left(\lambda_{1}^{*}+\omega\right) \\
& \times\left[4 L \cosh \left(\theta_{1}+\theta_{2}^{*}\right)+2\left(L^{2}+M^{2}+1\right) \cosh \left(\theta_{1}+\theta_{1}^{*}\right)\right], \\
G_{3}= & i p_{c}\left(\lambda_{1}-\lambda_{1}^{*}\right)\left[\sigma_{1}^{*} \sigma_{2} \mu+\sigma_{1} \sigma_{2}^{*} \mu^{*}\right] \\
& +\eta_{c}\left[\left(\lambda_{1}+\omega\right)\left(\lambda_{1}^{*}+\omega\right)\left(\sigma_{1} \sigma_{1}^{*}+\sigma_{2} \sigma_{2}^{*}\right)^{2}\right. \\
& \left.+2 \sigma_{1} \sigma_{1}^{*} \sigma_{2} \sigma_{2}^{*}\left(\lambda_{1}-\lambda_{1}^{*}\right)^{2}\right],
\end{aligned}
$$

with

$$
\begin{aligned}
& \sigma_{1}=e^{\theta_{1}}+e^{\theta_{2}}(L+i M), \quad \sigma_{2}=e^{\theta_{2}}+e^{\theta_{1}}(L+i M), \\
& \mu=\sigma_{1} \sigma_{1}^{*}\left(\lambda_{1}+\omega\right)+\sigma_{2} \sigma_{2}^{*}\left(\lambda_{1}^{*}+\omega\right) .
\end{aligned}
$$

Now, we mainly discuss soliton solutions from three different cases.

Case 1. In the case of $q_{c}=0$, that is to say, the initial seeds for (4a), (4b), and (4c) are $q_{c}=0, p_{c}=0$, and $\eta_{c}=1$, solutions $(22 \mathrm{a}),(22 \mathrm{~b})$, and $(22 \mathrm{c})$ reduce to one-soliton solutions.

Case 2. In the case of $q_{c} \neq 0, A_{s}=\omega_{c}=0$, and $\kappa_{s}^{2}>4 q_{c}^{2}$, symbolic computation results in the following:

$$
\begin{aligned}
& \zeta_{1}=\sqrt{-4 q_{c}^{2}+\kappa_{s}^{2}}, \quad \eta_{1}=0, \\
& \zeta_{2}=\frac{\zeta_{1} h_{1}}{4 \omega^{2}+\kappa_{s}^{2}}, \quad \eta_{2}=\frac{\zeta_{1} h_{2}}{4 \omega^{2}+\kappa_{s}^{2}},
\end{aligned}
$$




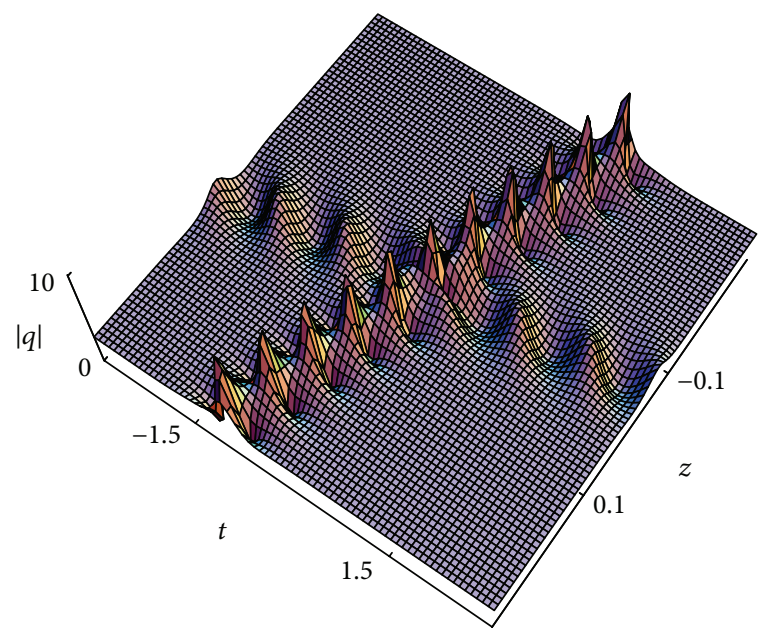

(a)

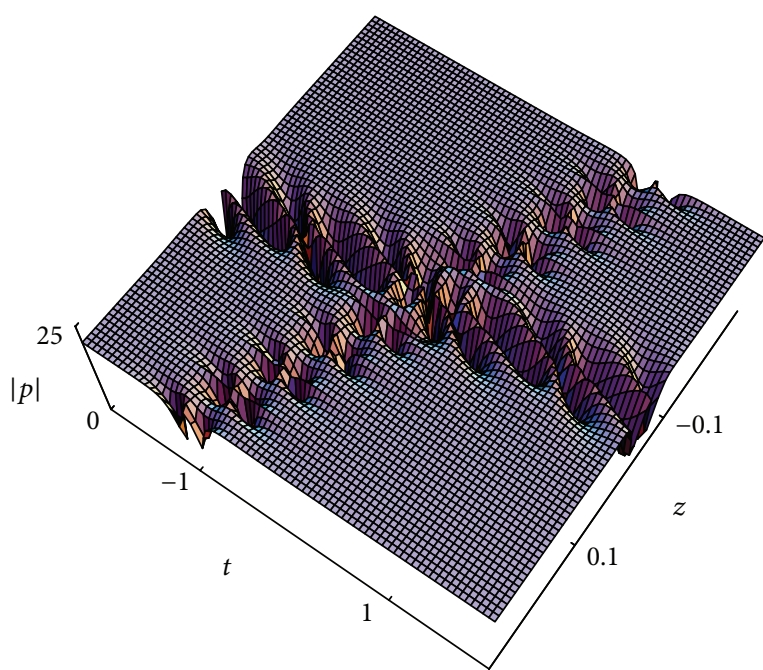

(b)

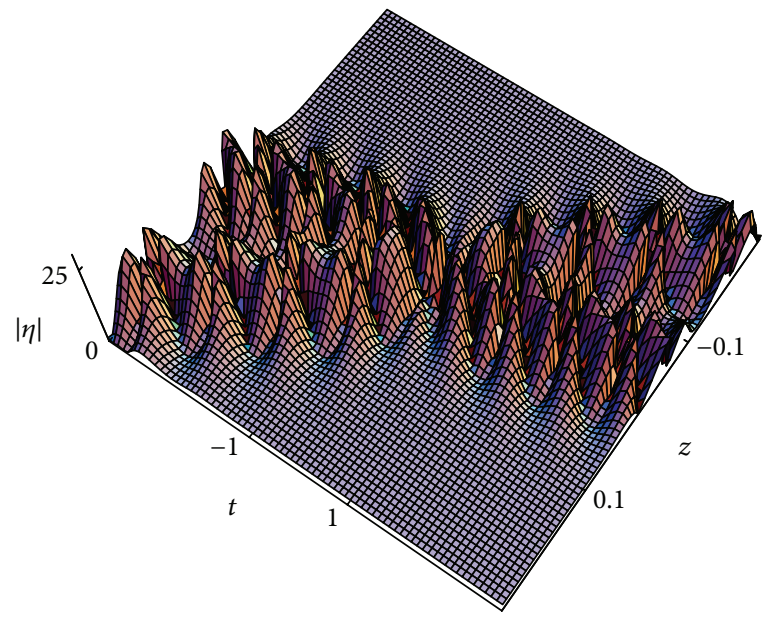

(c)

Figure 8: The parameters adopted here are $\lambda_{1}=i, \lambda_{2}=2 i, q_{c}=3, \omega_{c}=2, \kappa_{c}=1, \omega=0.5$, and $\tau=0.1$.

with

$$
\begin{gathered}
h_{1}=2 q_{c}^{2}-\kappa_{c}+\kappa_{s}^{2}+6 \tau q_{c}^{4}+2 \tau q_{c}^{2} \kappa_{s}^{2}+\tau \kappa_{s}^{4}, \\
h_{2}=-2 \omega \kappa_{s}-4 \omega \tau \kappa_{s} q_{c}^{2}-2 \omega \tau \kappa_{s}^{3} .
\end{gathered}
$$

Substituting (25) into (17a) and (17b), we can reduce solutions (22a), (22b), and (22c) to the breathers displayed in Figures 1 and 2 .

From Figure 1, we can observe that the main features of propagations of those breathers- are periodic in the space coordinate and aperiodic in the time coordinate, so the solitons shown in Figure 1 are Akhmediev breathers [21-23]. In addition, we can observe that Figures 1(a) and 1(c) depict bright breathers, and Figure 1(b) shows the dark one.

Comparing Figure 2 with Figure 1, one can see that in Figure 2, under the influence of the increasing values of the parameter $\tau$, the number of peaks on the same space interval is increasing when $\tau$ goes up from 0 to 0.1 . So, we can conclude that the parameter $\tau$ controls the period of the Akhmediev breathers.

Case 3. In the case of $q_{c} \neq 0, A_{s}=\omega_{c}=0$, and $\kappa_{s}^{2}<4 q_{c}^{2}$, through direct computation, one can obtain the following:

$$
\begin{aligned}
& \zeta_{1}=0, \quad \eta_{1}=\sqrt{4 q_{c}^{2}-\kappa_{s}^{2}}, \\
& \zeta_{2}=\eta_{1} \frac{h_{1} \kappa_{s}-2 h_{2} \omega}{4 \omega^{2}+\kappa_{s}^{2}}, \quad \eta_{2}=\eta_{1} \frac{2 h_{1} \omega+h_{2} \kappa_{s}}{4 \omega^{2}+\kappa_{s}^{2}} .
\end{aligned}
$$

Substituting (27) into (17a) and (17b), solutions (22a), (22b), and $(22 \mathrm{c})$ become other breathers as displayed in Figure 3.

From Figure 3, one can observe that those breathers are periodic in the time coordinate and aperiodic in the space coordinate; that is, those are Ma-breather solitons [24-27], and for functions $q$ and $\eta$, the solutions are bright solitons while for function $p$ the solution is the dark one. In addition, one can find that the separations between adjacent peaks 


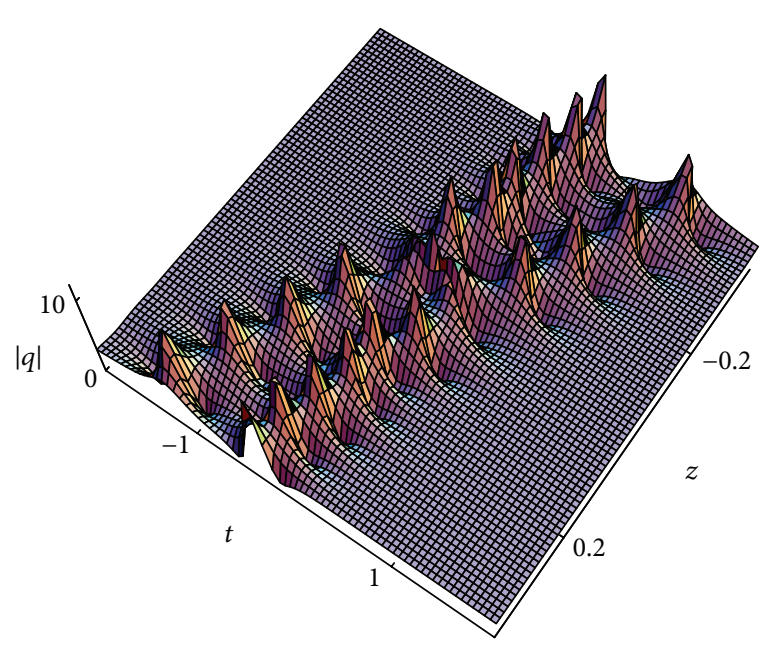

(a)

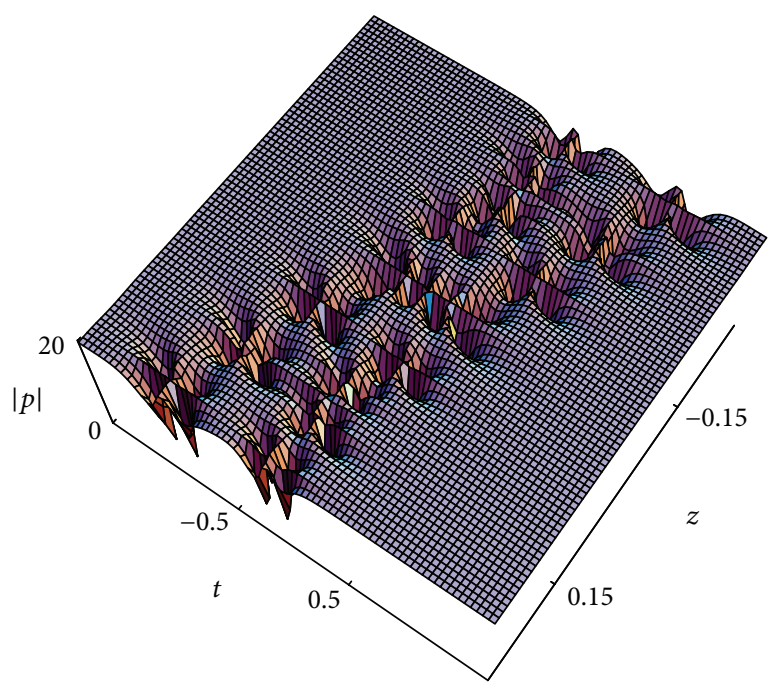

(b)

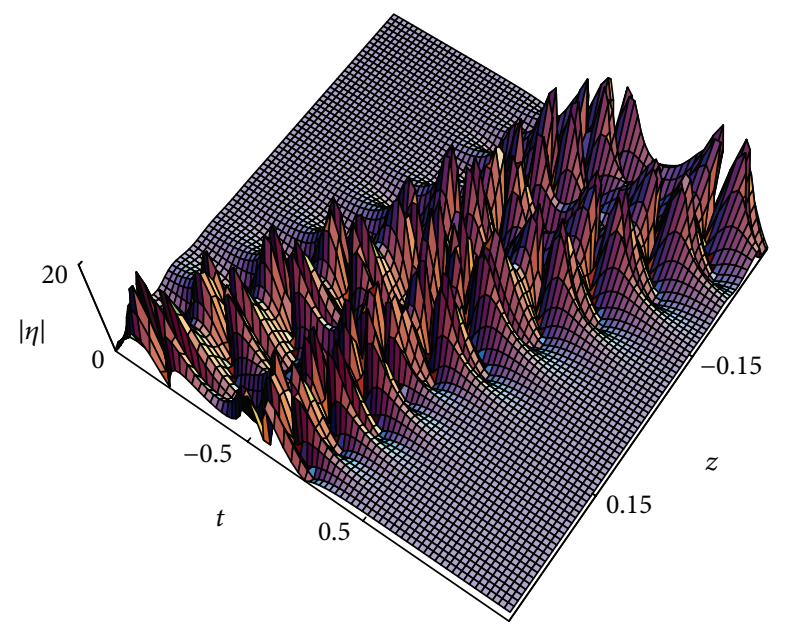

(c)

FIgURE 9: The parameters adopted here are $\lambda_{1}=5 i, \lambda_{2}=4 i, q_{c}=3, \omega_{c}=2, \kappa_{c}=1, \omega=0.5$, and $\tau=0.1$.

in Figure 3 gradually increase as $\kappa_{s} \rightarrow 2 q_{c}$ and eventually reduce into the rogue waves, the properties of which have been discussed in [28-31].

\section{Dynamic Features of Two-Soliton Solutions for System (4a), (4b), and (4c)}

In this section, we will construct two-soliton solutions for system (4a), (4b), and (4c). Taking the same seeds in Section 2, that is, $q=q_{c} \exp i\left(\kappa_{c} z+\omega_{c} t\right), p=i p_{c} \exp i\left(\kappa_{c} z+\omega_{c} t\right), \eta=\eta_{c}$, and iterating the DT twice, one can obtain

$$
\left(\begin{array}{l}
\psi_{1} \\
\psi_{2}
\end{array}\right)=\left[\lambda_{2}\left(\begin{array}{ll}
1 & 0 \\
0 & 1
\end{array}\right)-H \Lambda H^{-1}\right]\left(\begin{array}{l}
\varphi_{1} \\
\varphi_{2}
\end{array}\right)
$$

where 
with

$$
\begin{aligned}
\theta_{3}= & \frac{t}{2}\left[-i \omega_{c}+\sqrt{-\omega_{c}^{2}-4 q_{c}^{2}-4 \lambda_{2}^{2}-4 \lambda_{2} \omega_{c}}\right] \\
& +\frac{z}{2}\left[-i \kappa_{c}+\sqrt{-\kappa_{c}^{2}+4 a^{\prime 2}-4 b^{\prime 2}-4 i a^{\prime} \kappa_{c}}\right], \\
\theta_{4}= & \frac{t}{2}\left[-i \omega_{c}-\sqrt{-\omega_{c}^{2}-4 q_{c}^{2}-4 \lambda_{2}^{2}-4 \lambda_{2} \omega_{c}}\right] \\
& +\frac{z}{2}\left[-i \kappa_{c}-\sqrt{-\kappa_{c}^{2}+4 a^{\prime 2}-4 b^{\prime 2}-4 i a^{\prime} \kappa_{c}}\right], \\
a^{\prime}=2 i \lambda_{2}^{2}\left(-1+4 \lambda_{2}^{2} \tau\right)-i q_{c}^{2} & \times\left(-1+4 \lambda_{2}^{2} \tau-4 \lambda_{2} \omega_{c} \tau+3 \omega_{c}^{2} \tau\right)+3 i q_{c}^{4} \tau \\
& +\frac{i \eta_{c}}{\lambda_{2}+\omega}, \\
b^{\prime}= & q_{c}\left(4 \lambda_{2} q_{c}^{2} \tau+2 \lambda_{2}-\omega_{c}-4 \lambda_{2}^{2} \omega_{c} \tau\right. \\
& \left.\quad-2 \lambda_{2} \omega_{c}^{2} \tau+\omega_{c}^{3} \tau-8 \lambda_{2}^{3} \tau-6 q_{c}^{2} \omega_{c} \tau\right) \\
& +\frac{p_{c}}{\lambda_{2}+\omega},
\end{aligned}
$$

$\lambda_{2}$ is another eigenvalue for Lax pair (5a) and (5b), and $d_{1}, d_{2}$, $d_{3}$, and $d_{4}$ are complex constants satisfied as

$$
\begin{aligned}
d_{3}=\frac{1}{q_{c}} & {\left[i \omega_{c}+i \lambda_{2}+\frac{1}{2}\right.} \\
& \left.\times\left(-i \omega_{c}+\sqrt{-\omega_{c}^{2}-4 q_{c}^{2}-4 \lambda_{2}^{2}-4 \lambda_{2} \omega_{c}}\right)\right] d_{1},
\end{aligned}
$$

$d_{2}$

$$
\begin{aligned}
& =\frac{q_{c}}{(1 / 2)\left(-i \omega_{c}+\sqrt{-\omega_{c}^{2}-4 q_{c}^{2}-4 \lambda_{2}^{2}-4 \lambda_{2} \omega_{c}}\right)+i \omega_{c}+i \lambda_{2}} \\
& \quad \times d_{4} .
\end{aligned}
$$

Thus, one can derive the expressions of two-soliton solutions for system (4a), (4b), and (4c) as

$$
\begin{gathered}
q^{(2)}=q^{\prime}-2 i \frac{\left(\lambda_{2}-\lambda_{2}^{*}\right) \psi_{1} \psi_{2}^{*}}{\left|\psi_{1}\right|^{2}+\left|\psi_{2}\right|^{2}} \\
p^{(2)}=\frac{p^{\prime} \chi_{1}^{\prime 2}}{\chi_{2}^{\prime}\left(\left|\psi_{1}\right|^{2}+\left|\psi_{2}\right|^{2}\right)^{2}}-\frac{p^{\prime *}\left(\lambda_{2}-\lambda_{2}^{*}\right) \psi_{1}^{2} \psi_{2}^{* 2}}{\chi_{2}^{\prime}\left(\left|\psi_{1}\right|^{2}+\left|\psi_{2}\right|^{2}\right)^{2}} \\
+\frac{2 \eta^{\prime} \chi_{1}^{\prime}\left(\lambda_{2}-\lambda_{2}^{*}\right) \psi_{1} \psi_{2}^{*}}{\chi_{2}^{\prime}\left(\left|\psi_{1}\right|^{2}+\left|\psi_{2}\right|^{2}\right)^{2}} \\
\eta^{(2)}=\eta^{\prime}\left(1+\frac{2\left(\lambda_{2}-\lambda_{2}^{*}\right)\left|\psi_{1}\right|^{2}\left|\psi_{2}\right|^{2}}{\chi_{2}^{\prime}\left(\left|\psi_{1}\right|^{2}+\left|\psi_{2}\right|^{2}\right)^{2}}\right) \\
+\frac{p^{\prime}\left(\lambda_{2}-\lambda_{2}^{*}\right) \chi_{1}^{\prime} \psi_{2} \psi_{1}^{*}}{\chi_{2}^{\prime}\left(\left|\psi_{1}\right|^{2}+\left|\psi_{2}\right|^{2}\right)^{2}}-\frac{p^{\prime *}\left(\lambda_{2}-\lambda_{2}^{*}\right) \chi_{1}^{\prime *} \psi_{1} \psi_{2}^{*}}{\chi_{2}^{\prime}\left(\left|\psi_{1}\right|^{2}+\left|\psi_{2}\right|^{2}\right)^{2}}
\end{gathered}
$$

with

$$
\begin{aligned}
& \chi_{1}^{\prime}=\left(\lambda_{2}+\omega\right)\left|\psi_{1}\right|^{2}+\left(\lambda_{2}^{*}+\omega\right)\left|\psi_{2}\right|^{2}, \\
& \chi_{2}^{\prime}=\left(\lambda_{2}+\omega\right)\left(\lambda_{2}^{*}+\omega\right) .
\end{aligned}
$$

Now, we will analyze (32a), (32b), and (32c) under two different cases.

4.1. Interaction Characters of Bound Solitons. In this section, we will investigate the interaction between neighboring solitons for system (4a), (4b), and (4c). Taking $q_{c}=0, p_{c}=0$, $\eta_{c}=1$ and iterating the DT twice, one can generate twosoliton solutions with two spectral parameters $\lambda_{1}=\vartheta_{1}+i \varrho_{1}$ and $\lambda_{2}=\vartheta_{2}-i \varrho_{2}$ for system (4a), (4b), and (4c). Assuming the input launching pulses as $q(0, t)=\operatorname{sech}\left(t-t_{0}\right)+\operatorname{sech}\left(t+t_{0}\right)$, here $t_{0}$ denotes the soliton separation. Supposing that $\vartheta_{1}=$ $\vartheta_{2}=0$, we can derive that

$$
\varrho_{1,2}=1+\frac{2 t_{0}}{\sinh \left(2 t_{0}\right)} \pm \operatorname{sech} t_{0} .
$$

Under the circumstances $t_{0}=3$ and $\tau=0$, that is, the higherorder dispersive effects are absent, the two-soliton solutions can turn into bound solitons under suitable parameters chosen as shown in Figure 4. We can observe that main features are that the mutual attractions and repulsions repeat periodically when the bound solitons propagate, and this phenomenon may be harmful for optical soliton communication [32-34].

In fact we can suppress the periodical mutual attractions and repulsions through increasing the initial pulse separation, that is, the value of $t_{0}$. As portrayed in Figure 5, when $t_{0}=10$, one can find that the mutual attractions and repulsions between two bound solitons disappear, and the two solitons propagate in parallel without any effect on each other even if the propagation distance grows long enough. 
Figure 6 depicts the effects of the higher-order dispersive terms on the propagations of the solitons. As the pictures show, the periods of the bound solitons can be suppressed when $\tau$ decreases.

So we can conclude that the higher-order dispersive terms can control the propagation periods of the bound soliton.

4.2. Interaction Characters of Two-Breather Solutions. In this section, we will construct two-breather solutions for system (4a), (4b), and (4c). Taking $q_{c} \neq 0, p_{c} \neq 0, \eta_{c} \neq 0, \omega_{c}=0$ and iterating the DT twice, one can get two-breather solutions for system (4a), (4b), and (4c). When $\left|\lambda_{1}\right|<\left|q_{c}\right|$ and $\left|\lambda_{2}\right|>$ $\left|q_{c}\right|$, the Akhmediev breathers and Ma-breathers can coexist as portrayed in Figure 7. Main features of the interaction between two breathers in Figure 7 are that they interact perpendicularly and the shapes, amplitudes, and pulse widths of the two breathers all remain invariant, so the interactions are elastic.

When $\left|\lambda_{1}\right|<\left|q_{c}\right|$ and $\left|\lambda_{2}\right|<\left|q_{c}\right|$, the two-breather solutions that evolve from two Ma-breathers come into being as portrayed in Figure 8 . And the interactions between these two-breathers in Figure 8 are also elastic.

When $\left|\lambda_{1}\right|>\left|q_{c}\right|$ and $\left|\lambda_{2}\right|>\left|q_{c}\right|$, the two-breather solutions that evolve from two Akhmediev-breathers take place as depicted in Figure 9. And the interactions between these two-breathers in Figure 9 are also elastic.

\section{Conclusions}

Our main attention has been focused on system (4a), (4b), and (4c) which describes the propagation of optical solitons in nonlinear erbium-doped fibers with higher-order effects. Lax pair and modulation instability conditions for this system have been investigated. Two types of breathers (Akhmediev breathers and Ma-breathers), bound soliton solutions, and two-breather solutions have been constructed by Darboux transformation. Propagation properties of those solitons under the influences of higher-order effects have been discussed.

\section{Conflict of Interests}

The authors declare that there is no conflict of interests regarding the publication of this paper.

\section{Acknowledgments}

The authors express their sincere thanks to each member of their discussion group for their suggestions. This work has been supported by the Special Funds of the National Natural Science Foundation of China under Grant no. 11347165 and by Scientific and Technological Innovation Programs of Higher Education Institutions in Shanxi under Grant no. 2013110.

\section{References}

[1] M. J. Ablowitz, Solitons, Nonlinear Evolution Equations and Inverse Scattering, Cambridge University Press, Cambridge,
UK, 1992.

[2] G. P. Agrawal, Nonlinear Fiber Optics, Academic Press, San Diego, Calif, USA, 2001.

[3] M. J. Ablowitz, B. Prinari, and A. D. Trubatch, Discrete and Continuous Nonlinear Schrödinger Systems, Cambridge University Press, Cambridge, UK, 2003.

[4] A. Biswas, M. Fessak, S. Johnson et al., "Optical soliton perturbation in non-Kerr law media: traveling wave solution," Optics and Laser Technology, vol. 44, no. 1, pp. 263-268, 2012.

[5] K. Porsezian, M. Daniel, and M. Lakshmanan, "On the integrability aspects of the one-dimensional classical continuum isotropic biquadratic Heisenberg spin chain," Journal of Mathematical Physics, vol. 33, no. 5, pp. 1807-1816, 1992.

[6] H.-Q. Zhang, B. Tian, X.-H. Meng, X. Lü, and W.-J. Liu, "Conservation laws, soliton solutions and modulational instability for the higher-order dispersive nonlinear Schrödinger equation," The European Physical Journal B, vol. 72, no. 2, pp. 233-239, 2009.

[7] S. L. Palacios and J. M. Fernández-Díaz, "Black optical solitons for media with parabolic nonlinearity law in the presence of fourth order dispersion," Optics Communications, vol. 178, no. 4-6, pp. 457-460, 2000.

[8] M. Daniel, L. Kavitha, and R. Amuda, "Soliton spin excitations in an anisotropic Heisenberg ferromagnet with octupole-dipole interaction," Physical Review B: Condensed Matter and Materials Physics, vol. 59, no. 21, pp. 13774-13781, 1999.

[9] A. I. Maimistov and A. M. Basharov, Nonlinear Optical Waves, Springer, Berlin, Germany, 1999.

[10] K. Porsezian, "Optical solitons in some SIT type equations," Journal of Modern Optics, vol. 47, no. 10, pp. 1635-1644, 2000.

[11] K. Porsezian and K. Nakkeeran, "Solitons in random nonuniform erbium doped nonlinear fiber media," Physics Letters A, vol. 206, no. 3-4, pp. 183-186, 1995.

[12] J.-S. He, Y. Cheng, and Y.-S. Li, “The darboux transformation for NLS-MB equations," Communications in Theoretical Physics, vol. 38, no. 10, pp. 493-496, 2002.

[13] K. Porsezian and K. Nakkeeran, "Optical soliton propagation in a coupled system of the nonlinear Schrödinger equation and the Maxwell-Bloch equations," Journal of Modern Optics, vol. 42, no. 9, pp. 1953-1958, 1995.

[14] Y. S. Xue, B. Tian, H. Q. Zhang, W. J. Liu, R. Guo, and F. H. Qi, "Soliton-like solutions of the coupled Hirota-Maxwell-Bloch system in optical fibers with symbolic computation," Physica Scripta, vol. 79, no. 6, Article ID 065016, 2009.

[15] K. Porsezian and K. Nakkeeran, "Singularity structure analysis and the complete integrability of the higher order nonlinear Schrödinger-Maxwell-Bloch equations," Chaos, Solitons and Fractals, vol. 7, no. 3, pp. 377-382, 1996.

[16] C. Z. Li, J. S. He, and K. Porsezian, "Rogue waves of the Hirota and the Maxwell-Bloch equations," Physical Review E: Statistical, Nonlinear, and Soft Matter Physics, vol. 87, no. 1, Article ID 012913, 13 pages, 2013.

[17] M. J. Ablowitz, D. J. Kaup, A. C. Newell, and H. Segur, "Nonlinear-evolution equations of physical significance," Physical Review Letters, vol. 31, pp. 125-127, 1973.

[18] A. M. Kamchatnov, Nonlinear Periodic Waves and Their Modulations, World Scientific Publishing, Singapore, 2000.

[19] P. T. Dinda, C. M. Ngabireng, K. Porsezian, and B. Kalithasan, "Modulational instability in optical fibers with arbitrary higherorder dispersion and delayed Raman response," Optics Communications, vol. 266, no. 1, pp. 142-150, 2006. 
[20] O. C. Wright III, "Homoclinic connections of unstable plane waves of the long-wave-short-wave equations," Studies in Applied Mathematics, vol. 117, no. 1, pp. 71-93, 2006.

[21] N. N. Akhmediev and V. I. Korneev, "Modulation instability and periodic solutions of the nonlinear Schrödinger equation," Theoretical and Mathematical Physics, vol. 69, no. 2, pp. 10801093, 1986.

[22] R. Guo and H.-Q. Hao, "Breathers and multi-soliton solutions for the higher-order generalized nonlinear Schrödinger equation," Communications in Nonlinear Science and Numerical Simulation, vol. 18, no. 9, pp. 2426-2435, 2013.

[23] L. Li, Z. H. Li, S. Q. Li, and G. S. Zhou, "Modulation instability and solitons on a $\mathrm{cw}$ background in inhomogeneous optical fiber media," Optics Communications, vol. 234, no. 1-6, pp. 169176, 2004.

[24] Z. Y. Xu, L. Li, Z. H. Li, and G. S. Zhou, "Modulation instability and solitons on a cw background in an optical fiber with higherorder effects," Physical Review E: Statistical, Nonlinear, and Soft Matter Physics, vol. 67, no. 2, Article ID 026603, 7 pages, 2003.

[25] S. Q. Li, L. Li, Z. H. Li, and G. S. Zhou, "Properties of soliton solutions on a cw background in optical fibers with higherorder effects," Journal of the Optical Society of America B, vol. 21, no. 12, pp. 2089-2094, 2004.

[26] Y. C. Ma, "The perturbed plane-wave solutions of the cubic Schrödinger equation," Studies in Applied Mathematics, vol. 60, no. 1, pp. 43-58, 1979.

[27] G. Y. Yang, L. Li, and J. S. Tang, "Peregrine rogue waves induced by the interaction between a continuous wave and a soliton," Physical Review E: Statistical, Nonlinear, and Soft matter Physics, vol. 85, no. 4, Article ID 046608, 8 pages, 2003.

[28] J. S. He, H. R. Zhang, L. H. Wang, K. Porsezian, and A. S. Fokas, "Generating mechanism for higher-order rogue waves," Physical Review E: Statistical, Nonlinear, and Soft Matter Physics, vol. 87, no. 5, Article ID 052914, 10 pages, 2013.

[29] C. Z. Li, J. S. He, and K. Porsezian, "Nonlinear waves of the Hirota and the Maxwell-Bloch equations in nonlinear optics," Chinese Physics B, vol. 22, no. 4, Article ID 044208, 2013.

[30] J. S. He, S. W. Xu, and K. Porsezian, " $N$-order bright and dark rogue waves in a resonant erbium-doped fiber system," Physical Review E: Statistical, Nonlinear, and Soft Matter Physics, vol. 86, no. 6, Article ID 066603, 17 pages, 2012.

[31] J. S. He, S. W. Xu, and K. Porsezian, "New types of rogue wave in an erbium-doped fibre system," Journal of the Physical Society of Japan, vol. 81, no. 3, Article ID 033002, 4 pages, 2012.

[32] Z. Y. Xu, L. Li, Z. H. Li, and G. S. Zhou, "Soliton interaction under the influence of higher-order effects," Optics Communications, vol. 210, no. 3-6, pp. 375-384, 2002.

[33] R. Y. Hao, L. Li, Z. H. Li, W. R. Xue, and G. S. Zhou, "A new approach to exact soliton solutions and soliton interaction for the nonlinear Schrödinger equation with variable coefficients," Optics Communications, vol. 236, no. 1-3, pp. 79-86, 2004.

[34] R. C. Yang, X. L. Wu, R. Y. Hao, L. Li, and G. S. Zhou, "Interactions between neighboring combined solitary waves," Optics Communications, vol. 276, no. 1, pp. 149-157, 2007. 


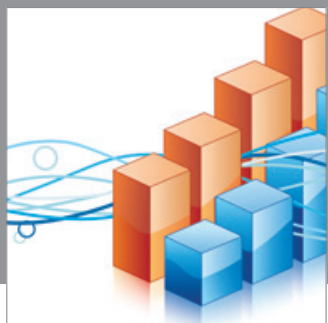

Advances in

Operations Research

mansans

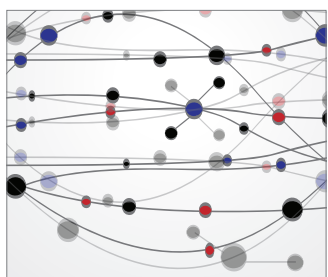

The Scientific World Journal
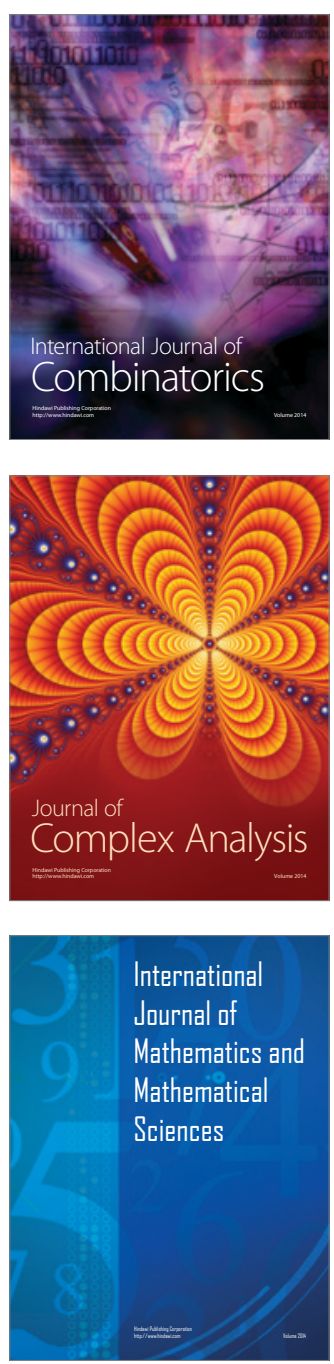
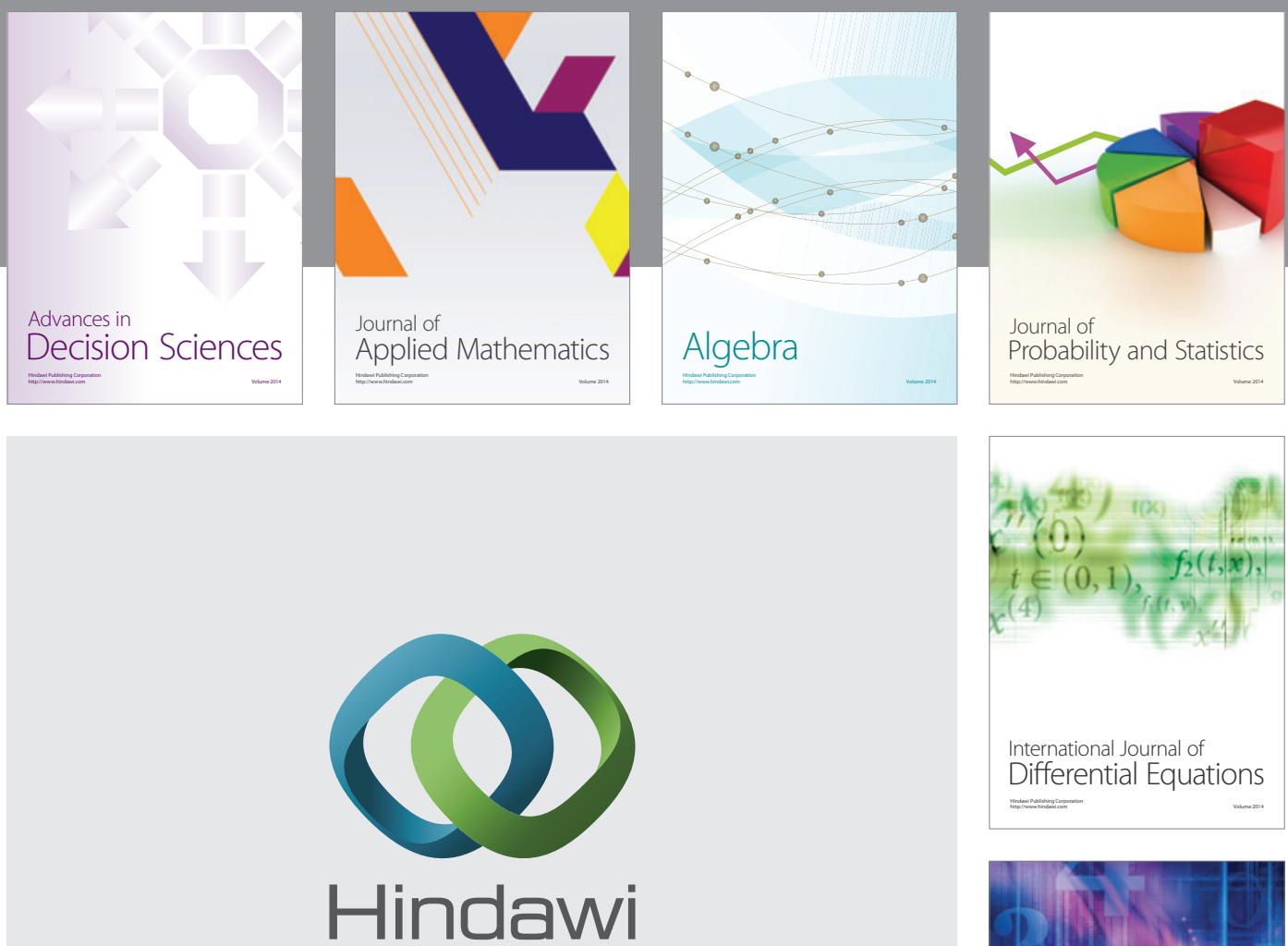

Submit your manuscripts at http://www.hindawi.com
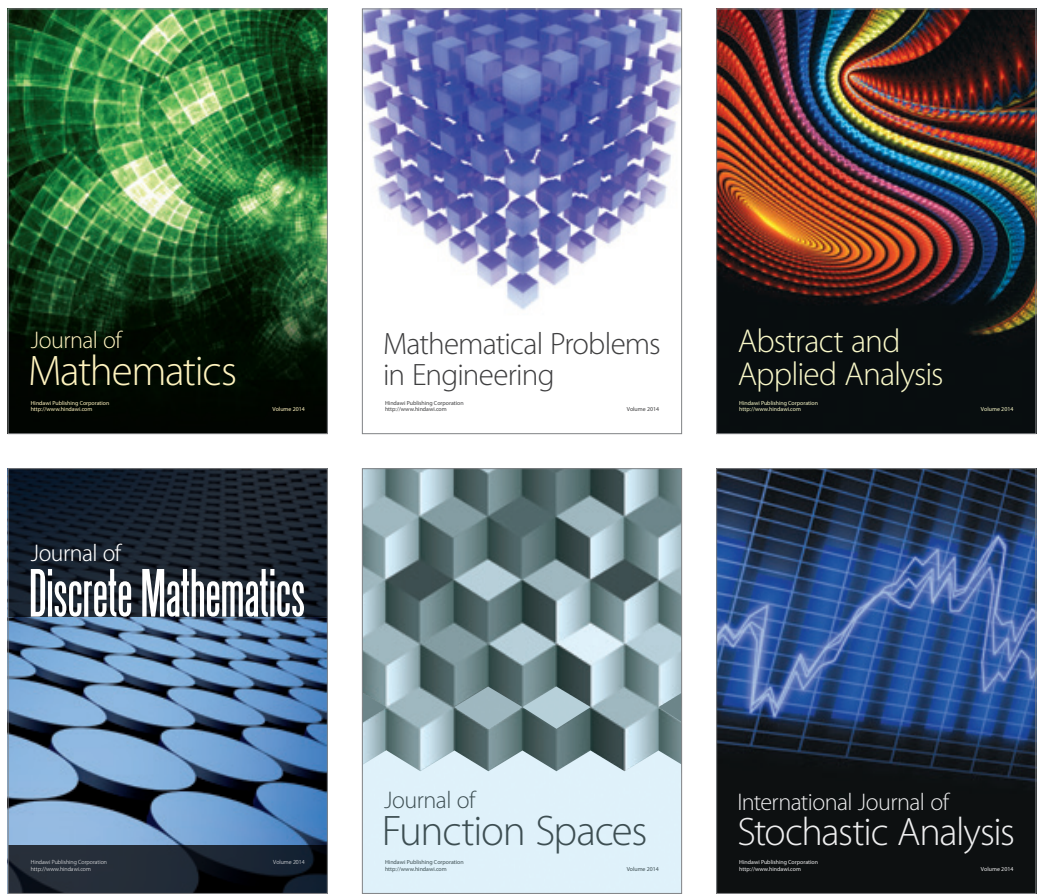

Journal of

Function Spaces

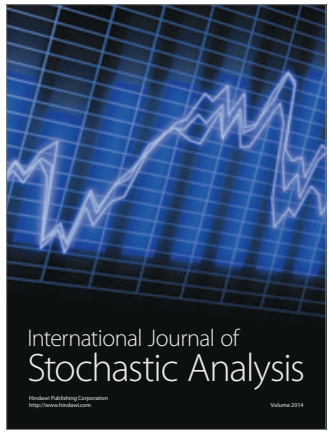

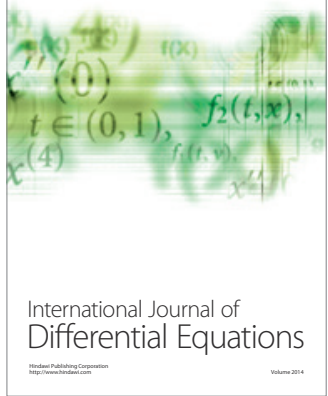
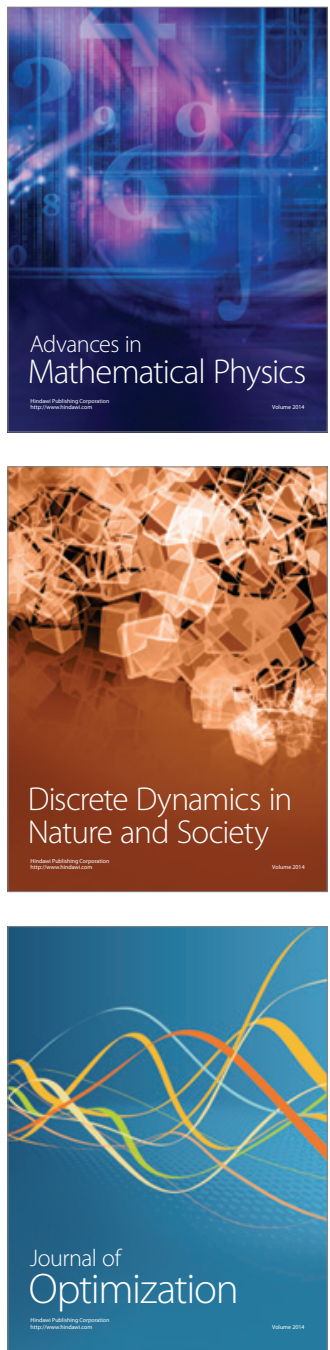